\title{
Artificial Sweeteners as a Cause of Obesity: Weight Gain Mechanisms and Current Evidence
}

\author{
Thiago Magalhães Cabral' ${ }^{1}$, Maira Guimarães Barboza Pereira ${ }^{1}$, \\ Amanda Emiko Zensque Falchione1, Diana Aristotelis Rocha de Sá1, Luciana Correa1, \\ Daniela da Maia Fernandes' ${ }^{1}$, Larissa Bianca Paiva Cunha de Sá1, Alberto Krayyem Arbex ${ }^{1,2,3}$ \\ ${ }^{1}$ Endocrinology Division, IPEMED Medical School, São Paulo, Brazil \\ ${ }^{2}$ Diabetology Department, Malteser Krankenhaus St.-Franziskus Hospital, Flensburg, Germany \\ ${ }^{3}$ Harvard T. H. Chan School of Public Health, Boston, MA, USA \\ Email: contato@drthiagocabral.com.br,dramairaguimaraes@gmail.com, am andafalchione@hotmail.com
}

How to cite this paper: Cabral, T.M. Pereira, M.G.B., Falchione, A.E.Z., de Sá, D.A.R., Correa, L., da Maia Fernandes, D., de Sá, L.B.P.C. and Arbex, A.K. (2018) Artificial Sweeteners as a Cause of Obesity: Weight Gain Mechanisms and Current Evidence. Health, 10, 700-717.

https://doi.org/10.4236/health.2018.105054

Received: March 21, 2018

Accepted: May 27, 2018

Published: May 30, 2018

Copyright $(9) 2018$ by authors and Scientific Research Publishing Inc. This work is licensed under the Creative Commons Attribution International License (CC BY 4.0).

http://creativecommons.org/licenses/by/4.0/

\begin{abstract}
The obesity epidemic gathers growing media attention recently, as overweight and obesity's prevalence keeps rising. This comes along with an increase in the intake of artificial sweeteners in food products. A causal relationship between the consumption of sweeteners and obesity is so far not clear in the medical literature. This paper describes the development of artificial sweeteners in a historical context. It collects epidemiological and experimental evidence that possibly relates the use of artificial sweeteners with weight gain. Finally, these effects are explained based on the neuroscience of food reward, the possible effects of glucose on the metabolism and the association between sweeteners and gut microbiota.
\end{abstract}

Keywords

Sweeteners, Weight Gain, Obesity, Microbiota, Cyclamate, Sucralose

\section{Introduction}

Obesity has become a global epidemic. In the United States, 30\% of adults are obese and $50 \%$ of children are overweight [1]. The large number of incidences increases medical expenses, which are at US $\$ 147$ billion annually in the United States [2] and US $\$ 81$ billion in the European Union [3]. These costs tend to grow, considering the prevalence of the disabled has increased over the years. In addition, this decreases quality of life, influencing mobility and mortality. Nowadays, some children have a lower life expectancy than their parents [4]. Obesity is a multifactorial disease, involving not only genetic, but also environmental, 
neuro-behavioral, endocrine and epigenetic causes [5].

To take on this complex universe, the US government has made many attempts to try to prevent obesity in all age groups in recent years. One example is the Dietary Guidelines Advisory Committee, which encourages adults and children to reduce their sugar consumption to less than $10 \%$ of total calories/day, and to reach a desirable goal of $<5 \%$ of refined sugars energy intake in both populations [6].

Many options have come as an alternative to losing or maintaining weight. There are people selling "fad diets" everywhere, and the scientific community has been struggling to find the most healthy, consistent and practical way to maintain body weight. In a survey, 186 people were randomized into two groups: one with less than 40 grams of carbohydrate, and the other with $30 \%$ of calories coming from fats, in a RCT (Randomized Controlled Trial). After 12 weeks, the low carb diet resulted in 3 times more weight loss [7]. However, in another Canadian study, a head-to-head trial with a 12-week follow-up comparing Atkins and South Beach low-carb diets showed no difference in outcome [8]. Thus, researchers found that weight loss is similar by comparing the diets.

These studies concluded that, in diets with the goal of weight loss in the adult population, changing the dietary components results in subtle weight loss; weight loss is more successful when the dietary energetic amount is restricted. But, among the restrictions, those diets with lower carbohydrate intake appear to be more promising.

If both traditional and fad diets led to the same weight loss, this would create a need to replace sugar. Sweeteners have become a tool to fight obesity, since they have a palatable sweetness and are almost calorie-free.

Less than 30 years ago, sweeteners were used only for diabetic patients or patients on doctor-recommended sugar restriction. With the media explosion and cult to the "perfect body", these substances have come to be used as substitutes for conventional sugar; at the same time, the obesity epidemic around the world has grown significantly. So, this epidemic coincides with an increase in the use of artificial sweeteners. Among the most popular sweeteners are Aspartame and Sucralose.

In this study, we investigate whether there is a causal relationship between the obesity epidemic and the increase in artificial sweeteners intake.

Increasing weight gain, and concerns about obesity, diabetes and cardiovascular disease, have influenced the use of artificial sweeteners in place of common sugar. Until the 1980s there were only 3 types of artificial sweeteners available: saccharin, cyclamate and aspartame, known as the first-generation sweeteners [9].

Since then, a second generation of sweeteners, represented by sucralose and acesulfame- $\mathrm{K}$, has been approved for human consumption and are gradually conquering the market worldwide. Some sweeteners, such as alitame, stevia, neohesperidin, neotame and thaumatin, are marketed and consumed only in some countries or geographic regions and are less known [10]. 


\section{Types of Sweeteners}

\subsection{Saccharin}

Saccharin was the first artificial sweetener, which started being marketed in the US in 1901. It was widely used during the last two world wars due to sugar supply problems.

Discovered accidentally in 1879 , saccharin is a derivative of naphthalene with a sweetening ability 400 times higher than sugar. It is absorbed slowly by the gastrointestinal tract and rapidly and thoroughly excreted by the kidneys [11]. When used in very high concentrations, the sweetener has a bitter taste, so it has been associated with other sweeteners since 1950, when cyclamate was discovered [9].

Some studies in animal models suggest that saccharin, even at optimal doses, may alter glucose tolerance in the gut, even when used for only 5 weeks [12]. Figure 1 shows the formule of Saccharin.

\subsection{Cyclamate}

Cyclamate has been marketed since 1950 and was developed by the researcher Michael Sweda. Chemically, it consists of cyclohexylsulfamic acid and sodium, calcium and potassium salts. It sweetens 30 to 140 times more than sugar and has no calories [1]. In 1970 it was banned from the USA for its carcinogenic potential, observed in some studies with animal models. Despite this, it is still marketed in more than 50 countries [2]. Figure 2 shows the formule of Cyclamate.

\subsection{Aspartame}

The sweet taste of Aspartame was discovered accidentally by James M. Schlatter in 1965 while working with the GD Searle laboratory on a substance that would be a gastrin inhibitor that would be used for treatment of peptic ulcer [3]. Thus, recognizing the compound's sweetening power, the laboratory conducted clinical animal trials during the 1960s and 1970s. In 1973, GD Searle petitioned the FDA for approval of aspartame as an artificial sweetener for certain foods. In 1974 it finally was approved by the FDA for certain foodstuffs [4].<smiles>O=C1NS(=O)(=O)c2ccccc21</smiles>

Figure 1. Saccharin molecule.

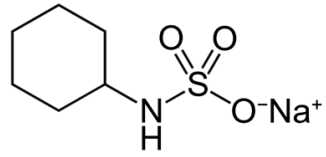

Figure 2. Cyclamate molecule. 
Aspartame is very similar to sugar and has the same caloric value ( $4 \mathrm{Kcal} / \mathrm{g}$ ), but its sweetening power is 180 - 200 times higher than sugar, which makes it useful as a sweetener. Chemically, it is a 2-aminoacid ester: aspartic acid and phenylalanine [5]. In the gut, it is hydrolyzed in aspartate, phenylalanine and methanol [6]. It has been reported that consumption of aspartame could cause neurological and behavioural disturbances in sensitive individuals [7]. Ingestion of aspartame results in a craving for carbohydrates, which will eventually result in weight gain, especially because the formaldehyde stores in the fat cells, particularly in the hips and thighs; therefore, aspartame is believed to cause problem in diabetic control [8]. Figure 3 shows the formule of Aspartame.

\subsection{Neotame}

Neotame is a new high-potency sweetener, considered a possible successor to aspartame. Monsanto purchased GD Searle Laboratory, which later became NutraSweet, in 1984. With the patent for aspartame broken in 1992, and the great competition with similar products, the company developed Neotame, a derivative of aspartame, with the addition of a group 3,3-dimethylbutyl to the free amine group of aspartic acid, which potentiates the sweetening power of the new molecule, which sweetens about 6000 to 10,000 times more than sucrose, becoming the sweetener with the highest potency ever developed. It has a good safety profile and is stable at high or low temperatures [9]. Studies show changes in body composition with the use of Neotame, such as long-term weight loss and increased consumption of unsweetened foods. However, these effects are not related to the toxic effects of the sweetener, but to the increase of sweetener concentrations in the food, which discreetly reduces palatability [10]. The Safety Studies found no toxicities, adverse effects or increased morbidity or mortality. Therefore, it is a relatively safe sweetener for consumption, according to the evidences [10]. Figure 4 shows the formule of Neotame.<smiles>COC(=O)[C@H](Cc1ccccc1)NC(=O)[C@H](N)CC(=O)O</smiles>

Figure 3. Aspartame molecule.

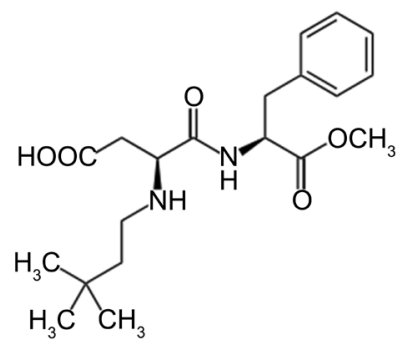

Figure 4. Neotame molecule. 


\subsection{Sucralose}

Sucralose is a disaccharide, 600 times sweeter than sucrose. It is obtained from the substitution of three groups of hydroxyls by three chlorine groups. It has no nutritional value, since it is practically not absorbed by the body, mostly excreted unchanged in the feces, and the little that is absorbed, is excreted intact in the urine [1].

It was discovered accidentally in 1976 by researchers from the British Sugar Company Tate \& Lyte, at the University of London, who researched applications of sucrose as an intermediary for the synthesis of other products. Several human studies support the view that sucralose does not alter glucose metabolism in the gut and is safe for patients with type 2 diabetes. However, in animal models, scientists have shown that consumption of sucralose in usual doses is able to reduce the beneficial fecal microbiota, increase fecal $\mathrm{pH}$, and increase the expression of P-gp, CYP3A4 and CYP2D1, which are known to limit the bioavailability of oral medications [11]. ${ }^{*} \mathrm{~A}$ recent study challenges the thermal stability of Sucralose. Oliveira et al. demonstrated that when heated, sucralose becomes chemically unstable, releasing chlorinated aromatic polycyclic hydrocarbons (CI-PAHs), toxic compounds, cumulative in the human body and potentially carcinogenic. CI-PAHs are associated with increased incidence of various types of cancers in humans. Figure 5 shows the formule of Sucralose.

\subsection{Acesulfame-K}

Acesulfame was discovered accidentally in 1967 by Clauss and Jensen. It is a synthetic potassium salt, derived from acetic acid, with sweetening power about 200 times higher than sucrose. This sweetener has a thermal stability that enables it to be put in fire without losing its properties [12]. Pharmacokinetic studies show that $95 \%$ of the consumed sweetener is excreted unchanged in the urine [1]. It has a pleasant taste, with the sweetness disappearing quickly, leaving a bitter taste at the end. This taste may be related to the activation of receptors of the TAS2R family, present in the taste buds that depending on the concentration, can stimulate the bitter taste [13]. Some studies suggest that Acesulfame- $\mathrm{K}$ may increase the absorption of glucose in the gut by activating enterocyte receptors to translocate GLUT2 to the membrane via the $\beta$ II PLC pathway, but this was confirmed only in high concentrations of the substance [14]. Figure 6 shows the formule of Acesulfame.

\subsection{Stevia}

Stevia is a generic name used to designate foodstuffs derived from the Stevia Rebaudiana Bertoni plant, which was used by the Guarani indigenous people, native to the border region between Paraguay and Mato Grosso do Sul (Brazil). Ovídio Rebaudi, a Paraguayan chemist, in his research, isolated the sweet compound from this plant in 1900. Seventy years later, this compound called Stevia Rebaudiana Bertoni was researched by Japanese scientists who began commercial extraction in 1995, after toxicological studies. It is considered a food 


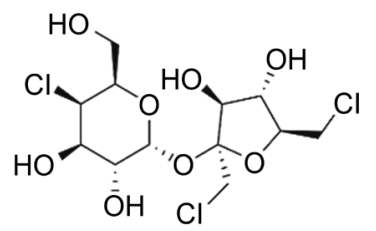

Figure 5. Sucralose molecule.<smiles>[Y]N1C(=O)C=C(C)OS1(=O)=O</smiles>

Figure 6. Acesulfame-K molecule.

supplement, free of calories, with sweetening power about 300 times higher than sucrose. It is not metabolized and remains stable at low or high temperatures Fonte bibliográfica inválida especificada. In animal models, Stevia showed ability to improve insulin sensitivity [15]. In humans, Stevia was able to improve glucose tolerance, reduce insulin levels and postprandial glycemia in the participants [16], and it could be used to manage postprandial hyperglycemia, suggesting that Stevia may aid in the control of glucose in type 2 diabetics [17]. Another study has shown that the use of Stevia for 2 years can control blood pressure, reducing systolic blood pressure (SBP) by about 6.8 to $7.3 \mathrm{mmHg}$, and by 3.2 to $4.2 \mathrm{mmHg}$ at diastolic pressure (DAP), and it seems to reduce the development of left ventricular hypertrophy $(P<0.001)$ [18]. This double effect (antihypertensive and antihyperglycemic) makes Stevia particularly useful in the treatment of obese patients with metabolic syndrome. Figure 7 shows the formule of Stevia.

\subsection{Xylitol}

Xylitol is a polyol found in small amounts in plants, microorganisms and animal tissues. It is as sweet as sugar, but $40 \%$ less caloric, known by organic chemistry at least since 1890. It was originally made by French and German researchers who succeeded in isolating the molecule in the 1930s. In 1975, the Finnish company Finnish Sugar Co Ltd began producing Xylitol on an industrial scale [19]. It is possible that the reaction between Xylitol and Calcium plays a role in reversing dental cavities. Xylitol stabilizes the phosphate-calcium system in saliva, in part, mimicking the function of natural salivary peptides, thus increasing the absorption of calcium by the tooth and providing protection against cavities [20]. In the gut, Xylitol is absorbed much slower than glucose since there is no specific transport mechanism. Only about $1 / 3$ of the consumed Xylitol is absorbed by diffusion (independent mechanism of insulin release), generating large amounts of hepatic glycogen. The remainder is broken down by gut bacteria into short-chain fatty acids, which are then absorbed and used by the body [19]. Figure 8 shows the formule of Xylitol. 


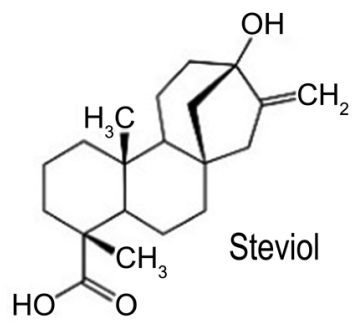

Figure 7. Stevia molecule.<smiles>OC[C@H](O)[C@H](O)[C@H](O)CO</smiles>

Figure 8. Xylitol molecule.

Table 1 shows a comparison of the main features of the studied sweeteners.

\section{Effects of Sweeteners Based on Neurobiology and Food Reward}

Contrary to the many divergences regarding the impacts of different types of sweeteners and whether glycemic metabolism can lead to diabetes, obesity and/or steatosis, the field of neurobiology appears to have found signs and metabolic pathways that may add to those who believe that sweeteners increase weight. One of the hypotheses would be about food reward. What mechanisms regulate the desire to eat? What are the mechanisms involved in the brain reward system? There are numerous studies that mention the interference of sweeteners in the light of neurobiology in different manners.

The behavioral and neurochemical effects of sugar intake lead to questioning whether sugar is an addictive substance or that may lead to a form of chemical dependence. The answer seems plausible, since the brain reward mechanisms activated when we ingest sugar are the same activated with the use of illicit drugs. The neural adaptations found in animal models are enkephalin mRNA expression, release of opioids, dopamine and acetylcholine in the nucleus accumbens. This can translate into human conditions as eating disorders and obesity [21].

In humans, after the ingestion of sugar, taste buds located on the tongue send signals that stimulate the primary gustatory area in the brain, which is located at the transition between the parietal operculum and the insula cortex. There may be stimulation in several other cortical areas, such as the hippocampus, the parahippocampal gyrus and the superior temporal sulcus [22]. The mesolimbic reward system functions as a reward center where several chemical messengers, including serotonin, enkephalin, $\gamma$-aminobutyric acid (GABA), dopamine (DA), acetylcholine $(\mathrm{ACH})$, among others, work together to provide a release of DA on 
Table 1. Comparative table between different types of sweeteners.

\begin{tabular}{|c|c|c|c|c|c|c|}
\hline SWEETENER & $\begin{array}{c}\text { CHARA } \\
\text { CTERISTICS }\end{array}$ & MOLECULE & CONTRAINDICATION & CALORIES & $\begin{array}{c}\text { SWEETENIN } \\
\text { G POWER* }\end{array}$ & $\begin{array}{c}\text { MAXIMUM } \\
\text { DAILY } \\
\text { DOSE }\end{array}$ \\
\hline Saccharin & $\begin{array}{l}\text { Synthetic. Produced } \\
\text { through an oil } \\
\text { derivative, } \\
\text { naphthalene. }\end{array}$ & & $\begin{array}{c}\text { People with hypertension } \\
\text { (relative } \\
\text { contraindication) }\end{array}$ & Zero & $\approx 400 \mathrm{x}$ & $5 \mathrm{mg} / \mathrm{Kg}$ \\
\hline Cyclamate & $\begin{array}{l}\text { Synthetic. A compound } \\
\text { based on an oil } \\
\text { derivative. }\end{array}$ & & $\begin{array}{c}\text { People with hypertension } \\
\text { (relative } \\
\text { contraindication) }\end{array}$ & Zero & $\approx 30-140 x$ & $11 \mathrm{mg} / \mathrm{Kg}$ \\
\hline Aspartame & $\begin{array}{c}\text { Produced from } \\
\text { Phenylalanine, Aspartic } \\
\text { Acid and Methane. }\end{array}$ & & $\begin{array}{c}\text { Phenylketonuric } \\
\text { individuals, pregnant and } \\
\text { nursing women }\end{array}$ & $4 \mathrm{Kcal} / \mathrm{g}$ & $\approx 200 \mathrm{x}$ & $40 \mathrm{mg} / \mathrm{Kg}$ \\
\hline Neotame & $\begin{array}{c}\text { Derived from } \\
\text { aspartame. Basically } \\
\text { aspartame plus a } \\
\text { 3.3-dimethylbutyl } \\
\text { radical }\end{array}$ & & $\begin{array}{l}\text { There are no } \\
\text { contraindications }\end{array}$ & $4 \mathrm{Kcal} / \mathrm{g}$ & $\begin{array}{l}\approx 6.000- \\
10.000 \mathrm{x}\end{array}$ & $2 \mathrm{mg} / \mathrm{kg}$ \\
\hline Sucralose & $\begin{array}{l}\text { Derived from sugar, } \\
\text { where } 3 \text { hydroxyls are } \\
\text { substituted by } 3 \\
\text { chlorine atoms. }\end{array}$ & & $\begin{array}{l}\text { There are no } \\
\text { contraindications }\end{array}$ & Zero & $\approx 600 \mathrm{x}$ & $5 \mathrm{mg} / \mathrm{Kg}$ \\
\hline Acesulfame $K$ & $\begin{array}{c}\text { Synthetic potassium } \\
\text { salt derived from acetic } \\
\text { acid }\end{array}$ & & $\begin{array}{l}\text { Kidney disease, and } \\
\text { individuals who limit } \\
\text { their potassium intake }\end{array}$ & Zero & $\approx 200 \mathrm{x}$ & $15 \mathrm{mg} / \mathrm{Kg}$ \\
\hline Stevia & $\begin{array}{l}\text { Extracted from the } \\
\text { plant Stevia } \\
\text { rebaudiana, native of } \\
\text { Paraguay, Brazil and } \\
\text { Argentina }\end{array}$ & Stevi & $\begin{array}{l}\text { There are no } \\
\text { contraindications }\end{array}$ & Zero & $\approx 300 \mathrm{x}$ & $5.5 \mathrm{mg} / \mathrm{Kg}$ \\
\hline Xylitol & $\begin{array}{l}\text { Sugar alcohol obtained } \\
\text { from the } \\
\text { hydrogenation of } \\
\text { xylose (xylitol) }\end{array}$ & \begin{tabular}{r|c}
$\mathrm{CH}_{2} \mathrm{OH}$ \\
$\mathrm{H}-$ & $\mathrm{OH}$ \\
$\mathrm{HO}-$ & $\mathrm{H}$ \\
$\mathrm{H}-$ & $\mathrm{OH}$ \\
& $\mathrm{CH}_{2} \mathrm{OH}$
\end{tabular} & $\begin{array}{l}\text { When ingested in excess, } \\
\text { it can cause diarrhea and } \\
\text { loss of minerals }\end{array}$ & $4 \mathrm{Kcal} / \mathrm{g}$ & & 15 mg/Kg \\
\hline
\end{tabular}

* Sweetening power compared to common sugar (sucrose) in a gram-gram ratio.

the nucleus accumbens (NAc). This circuit is implicated in the pleasure triggered by natural rewards, such as foods, especially sweet foods, constituting the neural basis for phenomena related to addiction [23]. When we eat palatable or sweet food, the brain reward system also encodes the caloric value of the food. A 
study with rats showed that this signaling is independent of the taste signaling. Rats who had their sweet taste receptor silenced and were fed sucrose activated their brain rewards system based solely on the caloric value of the food [24].

Evidence shows that non-caloric artificial sweeteners do not activate the rewards system like natural sweeteners. The lack of caloric contribution eliminates the activation of hypothalamic nuclei. Artificial and natural sweeteners also activate the taste receptors differently. The sweet taste receptor is a two-receptor heterodimer coupled to G, T1R2 and T1R3 proteins [25]. At practical levels, the sugar intake was responsible for greater activation of the brain gustatory areas compared to the saccharin, in which the activation was less pronounced [26].

In a study with 18 young men, Black et al. demonstrated that when they consumed water sweetened with aspartame, they had increased subjective hunger and desire to eat, unlike the group that ingested the same amount of water with aspartame capsules. Aspartame also increased the subjective rating of hunger compared to glucose or water intake [27]. Thus, in this case, aspartame sweetener may have a paradoxical effect on appetite. Aspartame decouples the sweet taste receptor from the caloric properties of the food and can distort the information used by the regulatory mechanisms involved in the control of food intake. In addition, aspartame may have other effects on appetite mechanisms, since phenylalanine, a precursor of catecholamine neurotransmitters, may increase food intake through hypothalamic adrenoreceptors implicated in central appetite control. In a study with 4 volunteers who ingested aspartame and common sugar, the group that consumed aspartame had a greater subjective sensation of hunger and less satiety compared to the group that consumed sugar [28]. These findings suggest that the calories contained in natural sweeteners can contribute to maintaining the body's energy needs, thus avoiding the paradoxical increase in appetite.

In studies with humans, assessments are subjective, which may impair the quality of the evidence. But in animal models (rats), this paradoxical effect is very similar. A study in rats conducted by Swithers and Davidson in 2008 showed that animals can use the taste of sweet to predict the caloric content of foods, thus maintaining energy homeostasis. Eating sweet and non-caloric substances may impair this predictive relationship, leading to a compensatory increase in caloric intake or reduction in the rate of basal metabolism. This study concluded that dissociation between sweet taste and caloric content of foods using the artificial sweetener Saccharin resulted in increased caloric intake, increased body weight and increased adiposity, as well as reduced thermogenesis for sweet-tasting diets, thus showing that diets containing artificial sweeteners can lead to weight gain and obesity by interfering with natural physiological and homeostatic processes [29]. These studies propose a hypothesis: the dissociation between sweet taste and caloric content can lead to a compensatory increase in food consumption and a positive energy balance. Finally, artificial sweeteners, precisely because they are sweet, stimulate the preference for the taste, the desire and the dependence for sweet foods [30], favoring an increase in the consump- 
tion and consequently, weight gain.

\section{The Use of Sweeteners Considering the Importance of Gut Microbiota}

The gut flora is composed of about 100 billion bacteria, with an average of 100 different species in a stable environment. The function of the mammalian gut flora is to increase the bioavailability of energy by trying to absorb and convert energy from substrates that are not absorbed naturally by the animals [31]. Different parts of the bowels allocate different phyla or species [32]. Many factors may affect the stability of the microbiota environment. One anthropological model suggested to explain this species variability is the need to extract the maximum energy from fruits/leaves or game pieces. The possibility of having different species of flora according to the diet was an adaptive resource, not only to extract most of the energy, but also to save it, if necessary.

The gut microbiota is a complex environment and influences many functions such as nutrition, energy homeostasis and body control. The microbiota and its metabolites are involved in intestinal permeability, in the mucosal immune function, in intestinal motility and even in the enteric nervous system.

The main trigger for changes in the flora is the type of food ingested. For example, African children who used to eat grains and large amounts of fiber had microbiota colonies with gram-negative bacteria, to improve the absorption of macronutrients [33]. However, the same children exposed to a diet high in sugar and fat showed a significant change in their microbiota, increasing the Firmicutes and decreasing Bacterioides. With this change, not only did they gain weight, but also presented decreased glucose tolerance. The same change in microbiota is seen with the use of saccharin in animal and human models and in patients with diabetes.

To explain the reasons for this change, many researchers have reported the possible metabolic pathways that may justify weight gain. Suez delimited the host's succession, previous metabolic disease, and the impact of dysbiosis. $\mathrm{He}$ describes other factors that lead to imbalance between gram positive and negative flora bacteria, in addition to commensal bacteria such as Akkermansia muciniphila and some Lactobacillus both would be responsible for not only maintaining permeability between the tight junction between the cells but also leading to greater protection against a pathogenic invasion. If there is a change in permeability, there will be a response of the immune system that will create metabolic impacts on the absorption of carbohydrates and fibers.

This difference in the distribution of flora species can anticipate the possible impact of certain substances, such as artificial and natural sweeteners on metabolism, since some sweeteners like fructose or acesulfame-k act more in the small intestine and others, such as sucralose and stevia, would act more in the large intestine [32].

New research added details to the complexity of the incretin effect of the mi- 
crobiota and to the effect on obesity and insulin resistance. For example, Suez designed a study that showed details about microbiota interfering in glycemic metabolism at different weeks according to the type of sweetener employed. It is believed that this result comes from different metabolic pathways. After proving the impact of the use of the three main sweeteners "saccharin", sucralose and aspartame on glycemic metabolism with worsening of the glucose tolerance curves, he sampled the feces of these rats with alteration in the microbiota and applies them to previously healthy rats, germ free without prior intolerance, but after the application of the flora, they begin to present glycemic changes as well as their flora donors. In this way, it shows that the impact was directly related to the flora. At the end of the study, it elevates the animal-to-human model by placing the glucose-tolerant human microbiota in germ-free mice, and these mice begin to respond poorly to glucose. Leading to believe that the metabolic pathways used in rats and humans are the same [34].

There are numerous explained pathways, among them glycosylation pathway, ascorbate metabolism, bile metabolism, LPS biosynthesis/endotoxemia, bacterial chemotaxis and Spirulina metabolism.

Among them, the most commented in the literature are the LPS and bile acid pathways.

The first one describes LPS by the high number of gram-negative bacteria that have LPS, a protein that, depending on the permeability between gut cells, can enter the epithelium and cause immune reaction. Dysbiosis due to saccharin use is characterized by decreased production of butyrate, which elevates the intestinal $\mathrm{pH}$ producing the Clostridium specimen that exposes LPS and leaves greater permeability for many gram negative opportunistic pathogens, including Bacterioides.

The LPL or endotoxin cell wall component found and gram-negative bacteria that stimulate inflammatory responses through activation increase the b-endotoxin factor. This happens in chronic diseases. The LPS translocation thought for the intercellular pathway alters the intestinal permeability of health, alternating the tight junction and mucous layer. Lactobacillus probiotic prevents against permeability in humans, the intestinal epithelium prevents against alpha and gamma tumor necrosis. In addition, the LPS protein is associated with chylomicrons and clears bacterial toxins.

The other pathway is that of bile acids that are involved in glucose homeostasis and remain not completely understood, but may be related to the action of FXR Farnesoid, a receptor that activates the pancreas, playing a role in the transport of insulin and its secretion. There is also FXR activation of the liver, a fact that improves insulin sensitivity [35]. However, the sweetener worsens the FXR factor in the liver, worsening insulin sensitivity and paradoxically increases activation in the pancreas releasing more insulin and worsening insulin resistance.

The third pathway studied is the short-chain free fatty acid route with buty- 
rate, which predominantly lowers the luminal $\mathrm{pH}$, this is a route used by Aspartame that leads to impacts on hepatic steatosis.

Attempting to delineate a few biases from previous research, microbiota tends to return to normal after exposure. Thus, studies that prove that use for a short time or single exposure would have as a bias that the flora tries to reestablish itself to its original form. Another important detail that was added was that it is already known that the high-fat, high-carbohydrate diet known as the Western diet, alters the microbiota. Therefore, many researches gave equal fat-rich diets that by themselves would alter the microbiota, so when comparing the two samples, there would be no significant index of sweetener use, since every diet would alter the microbiota of the two groups, leading to similar or insignificant results.

Another detail that was not observed previously was that when pure substances are administered, they have little impact on glucose, TTGO and incretin hormones, since there are several published studies on this, using different concentrations. However, it was not known that the effect of sweeteners on glycemic metabolism acts with the use of glucose at the same time. It was found that sucralose 10 min before the tolerance test had a worse impact on glycemia and insulin than without adding sugar. One of the new hypotheses for this is that the sweetener could stimulate the sweet receptor TR1 TR3 in the mouth and the same receptor in the gut, leading to a slower sugar response.

Regarding the taste for sweets, there is a new research on the discovery of TR1TR3, a membrane protein that recognizes sweet taste in the mouth and at the intestinal level. The first hypothesis is that the sweeteners did not act on the receptor, and therefore would lead to a lower perception of the amount of food, leading to higher intakes. The possible explanation would be the peripheral and central alteration that the sweeteners could take to change the sensitivity to sweets.

However, as with animals fed with sweeteners in agriculture, it is known that when fed with sugar they were able to be weaned faster and gain weight. However, due to the price increase, they started with artificial sweeteners and noticed the maintenance of the weight gain even though there was not an increase in calories, since the sweetener does not have any calories. One of the theories is that the sweet taste would generate more "hedonic eating" pleasure, and thus increase intake.

However, it is now known that sweeteners stimulate the transcription of SLGT1 genes to produce satiety hormones, but there is a $3 \mathrm{x}$ increase in the glucose transporter that leads to a greater amount of glucose into the circulation, increasing insulin, affecting the metabolic syndrome due to increased glucose supply in the peripheral circulation [36].

Depending on the study, the connection between obesity and microbiota can be analyzed as causality or association. An example is the study by Winther, a cross-sectional study that concluded that the use of sweeteners was associated with an unhealthy lifestyle with unfavorable diet, increased energy intake, in- 
cluding sugar, and reduced vitamin intake. Therefore, the patients would have numerous factors that would contribute to the alteration in the microbiota, which were aggravated by the use of the sweeteners [37].

\section{Discussion}

As described, there are numerous research biases that cause discordant results regarding weight gain and the use of sweeteners. The main ones are summarized below:

1) Sweetener generalization

Literature reviews or conclusions that generalize sweeteners as a single category are incorrect. Sweeteners have different biochemical structures with different metabolizing pathways, with or without bacteriostatic power, and excretory pathways that, in themselves, prevent a generalizing result. As an example, the metabolism of sucralose happens in the large intestine, of aspartame in the small intestine, each influencing the flora differently; model flora using saccharin looks like the model flora of diabetes, and the model flora using sucralose looks like model of autoimmune diseases, with less commensal bacteria, less mucous and more pathogens.

2) Different susceptibility

It is wrong to consider that all individuals are the same. Even population studies present several biases that affect the study and are not taken into consideration, mainly in retrospective and prospective cohort studies. For example, prior exposure in pregnancy, pre-study flora, dietary habits explained through a questionnaire, considering that studies show that patients do not know they use sucralose as an additive, because they are intrinsic in foods and are not described in labels. So, comparing people who are exposed or not exposed to sucralose based on dietary questionnaires would be wrong, because everybody is exposed to it. Therefore, studies that analyze everyone as exposed to different degrees can prove that the higher the intake, the greater the risk of weight gain and diabetes (i.e., a longitudinal study in SÃO FRANCISCO on the elderly and diet soda, with a 10-year follow-up). Gender also interferes in metabolization; women use more sucralose and have the same effect on weight gain, and men use it less, but gain more weight. Therefore, there is a great inter-gender variation that has not been researched yet. The same goes for ethnicity: given the same amount of diet beverages, non-Hispanic and Asians suffer more when compared to Caucasians and Blacks.

3) Sweeteners: causality or association?

It is known that $36 \%$ of people who use sweeteners are obese, $23 \%$ overweight and $11 \%$ eutrophic [38], so it can seem there is an association: the person is obese, so they use diet products to lose weight. However, recent studies show that the relationship is causal due to the microbiota and impact on the primitive alimentary behavior and hormonal neuromodulation, with SGLT1, GLUT2, efferent and central nerve responses of the vagus nerve, with different search routes 
and food with hedonic pleasure.

4) Beginning of contact and frequency

The concept of previous contact has been used to prove weight gain in both animal models, with weaning from breastfeeding and introduction to food, as well as in humans, with pregnant women who drank soda, and children with higher BMI compared to those who did not drink soda either in the first year, in the 5 years and up to the seventh year of follow-up. For example, pregnant women who drink diet soda presented more sucralose in the placenta than in the maternal tissues, proving that it passes through the placenta. Some types of soda, and also higher concentrations, were found in fetal tissues, if there was a single exposure compared to multiple exposures, which would have greater clearance of the substance. In addition to the increased susceptibility in the elderly and children, this group should not use sweeteners as a tool for weight loss, according to a British study and a San Francisco study [39].

5) The importance of microbiota in weight gain and IR

The microbiota is important for weight maintenance, as shown by an experimental bypass model that proved that surgically transplanting the flora of thin would result in lower intake and, consequently, weight loss. Other examples are a study with rats given antibiotics and subsequent use of sweeteners, leading to dysbiosis and insulin resistance, the SUEZ study or even epigenetics of SGA newborns with sparing phenotype being more susceptible to obesity. All corroborate the importance of the microbiota and its impact on weight.

6) Alterations in microbiota simulate flora that is typical of diabetes and obesity

Changes in the genetic transcripts related to glucosidases can interfere in the metabolism of the host, all to generate more energy. Having FILO Bacteroides in rats elevates $110 \mathrm{kcal}$ regardless of the weight of the mouse. This shows the part played by microbiota in weight. Another important route is the bile acid pathway that sweeteners such as saccharin and aspartame increase production of butyrate which, when metabolized in the liver, causes decrease in saccharin and increase in the $\mathrm{pH}$ aspartame, leading to the imbalance between the gram +/- gradient of the bacteria that leads to the growth of firmicutes in saccharin, sucralose with Bacteroides, saccharin increases clostridium which decreases the junctions between cells, decreases lactobacilli that increases commensal bacteria, which change the intestinal $\mathrm{pH}$, increases the Bacteroides in the flora and the cycle feeds itself.

7) T1R3 receptor stimulus and compulsion

The T1R3 receptor, responsible for the sensitivity to sugar, is stimulated by the sweeteners by up-regulation, and not down-regulation, as was believed, leading to an increase in craving for sugar. The more sweeteners, the more the sweet taste is stimulated, since the sweetener mimics the taste and binds more strongly to the heterodyne, resulting in the need for increasing doses. Ingesting sugar or industrial mixed solutions generates slower peripheral responses to sugar by the receptor. 
8) T1R3 receptor: central and peripheric action

Some studies proved that sweeteners did not change the concentration of SGLT1 in biochemistry, but at the practical level, when eating sucralose and sugar (a mixture typical of Splenda) the signal is slowed down, leading to a decrease in the sensation of satiety, and an increase in intake, worsening in the confirmed incretin effect with worsening of TTGO with sweeteners and dextrose used for the test [40]. Although T1R3 is the sugar sensitizer both in the mouth and in the gut, studies have found that SGLT1 is the major food marker for satiety. In pure sweeteners, sucralose does not produce SGLT1, however, when mixed with sugar, it overstimulates the symporter of sugar and sodium, independent of the fact that increasing voltage by $3 x$ on the surface of the apical membrane results in absorption of $3 \mathrm{x}$ more sugar, leading to responses at both peripheral and central level [41].

9) Route of administration and incretin action

At the peripheral level, aspartame, when it is ingested through the mouth, stimulates T1R3, if through gastric probes, it does not. Therefore, the route of administration of the sweetener is important. Also, que amount of solid or liquid produces different satiety responses. Many studies still used this for sweeteners and could not mimic the results orally. Just as all primitive responses start and clash with the incoming T1R3 inflammation. For example, aspartame administered through the mouth initiates the dopamine in the contraction TGI, stimulating several peripheral nerves and posterior central, but there is a disruption between the physiological response imagined with the aspartame that simulated sugar with the caloric contribution, and SGLT1 generated in the mouth by the T1R3 and found effectively afterwards with T1R3 in the gut.

10) Different reward areas

At the Central level, other sucralose and saccharin sweeteners do not stimulate the hypothalamic area like sugar, but do stimulate the amygdala area of fight and flight and reward areas of "hedonic eating", causing inflammations typical of obesity, leading to a worse sugar response, "obesity inflamed hypothalamus" [42].

\section{Conclusions}

Sweeteners are not a single category of food supplement. They have different biochemical structures, with different routes of metabolization and absorption. Its individual effects are associated with several factors, such as previous exposure during pregnancy, intestinal flora before the study, dietary habits, gender, among others. There are differences in effects between women and men, which have not been explained in comparative studies.

Studies have indicated a possible causal relationship between the use of sweeteners and obesity: in fact, about $60 \%$ of people who use sweeteners are overweight, and the gut microbiota would be responsible for changes in early eating behavior and adjustments in hormonal neuromodulation through the molecules SGLT-1, GLUT-2, efferent responses of the vagus and central nerves, with 
food-seeking pathways for hedonic pleasure.

Saccharin and aspartame raise the production of butyrate in the body and lead to an increase in the number of Bacteroidetes in the gut. These bacteria are directly associated with weight gain. Sweeteners such as sucralose and saccharin do not stimulate the hypothalamic area. They have other reward areas. They lead to a typical inflammation of obesity, and worsening response to sugar intake.

More studies are needed to elucidate mechanisms of hunger, satiety, gut microbiota and weight regulation that are still poorly understood and are modified by the use of sweeteners.

\section{References}

[1] Ada Report (2004) Position of the American Dietetic Association: Use of Nutritive and Nonnutritive Sweeteners. Journal of the American Dietetic Association, 104, 255-275. https://doi.org/10.1016/j.jada.2003.12.001

[2] Natividade, D.P., Denise de Andrade Rodrigues, C.G. and da Silva Vieira, V. (2011) XENOBIOTICS: Frequency of Inclusion in the Diet of Artificial Sweeteners Especially Aspartame, Sodium Cyclamate and Sodium Saccharin-Possible Adverse Effects. Revista Praxis, 3.

[3] Stegink, L.D. (1987) The Aspartame Story: A Model for the Clinical Testing of a Food Additive. The American Journal of Clinical Nutrition, 46, 204-215. https://doi.org/10.1093/ajcn/46.1.204

[4] Sturtevant, F.M. (1985) Use of Aspartame in Pregnancy. International Journal of Fertility, 30, 85-87.

[5] Torloni, M.R., et al. (2007) The Use of Sweeteners in Pregnancy: An Analysis of Products Available in Brazil. Revista Brasileira de Ginecologia e Obstetricia, 29, 267-275.

[6] Butchko, H.H., et al. (2002) Aspartame: Review of Safety. Regulatory Toxicology and Pharmacology, 35, S1-S93. https://doi.org/10.1006/rtph.2002.1542

[7] Centers for Disease Control (CDC) (1984) Evaluation of Consumer Complaints Related to Aspartame Use. Morbidity and Mortality Weekly Report, 33, 605-607.

[8] Humphries, P., Pretorius, E. and Naude, H. (2008) Direct and Indirect Cellular Effects of Aspartame on the Brain. European Journal of Clinical Nutrition, 62, 451-462. https://doi.org/10.1038/sj.ejcn.1602866

[9] Nofre, C. and Tinti, J.-M. (2000) Neotame: Discovery, Properties, Utility. Food Chemistry, 69, 245-257. https://doi.org/10.1016/S0308-8146(99)00254-X

[10] Mayhew, D.A., Phil Comer, C. and Wayne Stargel, W. (2003) Food Consumption and Body Weight Changes with Neotame, a New Sweetener with Intense Taste: Differentiating Effects of Palatability from Toxicity in Dietary Safety Studies. Regulatory Toxicology and Pharmacology, 38, 124-143. https://doi.org/10.1016/S0273-2300(03)00074-6

[11] Abou-Donia, M.B., et al. (2008) Splenda Alters Gut Microflora and Increases Intestinal p-Glycoprotein and Cytochrome p-450 in Male Rats. Journal of Toxicology and Environmental Health $A, 71,1415-1429$.

[12] Von Rymon, L., Gert-Wolfhard and Hanger, L.Y. (2001) Alternative Sweeteners. Marcel Dekker, Inc., New York.

[13] Kuhn, C., et al. (2004) Bitter Taste Receptors for Saccharin and Acesulfame K. Journal of Neuroscience, 24, 10260-10265. 
https://doi.org/10.1523/JNEUROSCI.1225-04.2004

[14] Zheng, Y. and Sarr, M.G. (2013) Effect of the Artificial Sweetener, Acesulfame Potassium, a Sweet Taste Receptor Agonist, on Glucose Uptake in Small Intestinal Cell Lines. Journal of Gastrointestinal Surgery, 17, 153-158.

[15] Chang, J.-C., et al. (2005) Increase of Insulin Sensitivity by Stevioside in Fructose-Rich Chow-Fed Rats. Hormone and Metabolic Research, 37, 610-616.

[16] Curi, R., et al. (1986) Effect of Stevia Rebaudiana on Glucose Tolerance in Normal Adult Humans. Brazilian Journal of Medical and Biological Research, 19, 771-774.

[17] Anton, S.D., et al. (2010) Effects of Stevia, Aspartame, and Sucrose on Food Intake, Satiety, and Postprandial Glucose and Insulin Levels. Appetite, 55, 37-43.

[18] Hsieh, M.-H., et al. (2003) Efficacy and Tolerability of Oral Stevioside in Patients with Mild Essential Hypertension: A Two-Year, Randomized, Placebo-Controlled Study. Clinical Therapeutics, 25, 2797-808.

[19] Mäkinen, K.K. (2004) History, Safety, and Dental Properties of Xylitol.

[20] Mäkinen, K.K. (2011) Sugar Alcohol Sweeteners as Alternatives to Sugar with Special Consideration of Xylitol. Medical Principles and Practice, 20, 303-320.

[21] Avena, N.M., Rada, P. and Hoebel, B.G. (2008) Evidence for Sugar Addiction: Behavioral and Neurochemical Effects of Intermittent, Excessive Sugar Intake. Neuroscience \& Biobehavioral Reviews, 32, 20-39.

[22] Kobayakawa, T., et al. (1999) Spatio-Temporal Analysis of Cortical Activity Evoked by Gustatory Stimulation in Humans. Chemical Senses, 24, 201-209.

[23] Ribeiro, G. and Osvaldo, S. (2013) Food Reward: Mechanisms Involved and Implications for Obesity. Portuguese Journal of Endocrinology, Diabetes and Metabolism, 8, 1-7.

[24] Smeets, P.A.M., et al. (2005) Functional Magnetic Resonance Imaging of Human Hypothalamic Responses to Sweet Taste and Calories. The American Journal of Clinical Nutrition, 82, 1011-1016.

[25] Cui, M., et al. (2006) The Heterodimeric Sweet Taste Receptor Has Multiple Potential Ligand Binding Sites. Current Pharmaceutical Design, 12, 4591-4600.

[26] Haase, L., Cerf-Ducastel, B. and Murphy, C. (2009) Cortical Activation in Response to Pure Taste Stimuli during the Physiological States of Hunger and Satiety. Neuroimage, 44, 1008-1021.

[27] Black, R.M., Leiter, L.A. and Harvey Anderson, G. (1993) Consuming Aspartame with and without Taste: Differential Effects on Appetite and Food Intake of Young Adult Males. Physiology \& Behavior, 53, 459-466.

[28] Blundell, J.E. and Hill, A.J. (1986) Paradoxical Effects of an Intense Sweetener (Aspartame) on Appetite. The Lancet, 1, 1092-1093. https://doi.org/10.1016/S0140-6736(86)91352-8

[29] Swithers, S.E. and Davidson, T.L. (2008) A Role for Sweet Taste: Calorie Predictive Relations in Energy Regulation by Rats. Behavioral Neuroscience, 122, 161-173.

[30] Liem, D.G. and De Graaf, C. (2004) Sweet and Sour Preferences in Young Children and Adults: Role of Repeated Exposure. Physiology \& Behavior, 83, 421-429.

[31] Utzschneider, K.M., et al. (2016) Mechanisms Linking the Gut Microbiome and Glucose Metabolism. The Journal of Clinical Endocrinology \& Metabolism, 101, 1445-1454. https://doi.org/10.1210/jc.2015-4251

[32] Spencer, M., et al. (2016) Artificial Sweeteners: A Systematic Review and Primer for Gastroenterologists. Journal of Neurogastroenterology and Motility, 22, 168-180. 
[33] De Filippo, C., et al. (2010) Impact of Diet in Shaping Gut Microbiota Revealed by a Comparative Study in Children from Europe and Rural Africa. Proceedings of the National Academy of Sciences, 107, 14691-14696. https://doi.org/10.1073/pnas.1005963107

[34] Suez, J., et al. (2014) Artificial Sweeteners Induce Glucose Intolerance by Altering the Gut Microbiota. Nature, 514, 181-186. https://doi.org/10.1038/nature13793

[35] Parséus, A., et al. (2017) Microbiota-Induced Obesity Requires Farnesoid X Receptor. Gut, 66, 429-437. https://doi.org/10.1136/gutjnl-2015-310283

[36] Daly, K., Darby, A.C. and Shirazi-Beechey, S.P. (2016) Low Calorie Sweeteners and Gut Microbiota. Physiology \& Behavior, 164, 494-500. https://doi.org/10.1016/j.physbeh.2016.03.014

[37] Winther, R., Aasbrenn, M. and Farup, P.G. (2017) Intake of Non-Nutritive Sweeteners Is Associated with an Unhealthy Lifestyle: A Cross-Sectional Study in Subjects with Morbid Obesity. BMC Obesity, 4, 41.

[38] Pepino, M.Y. (2015) Metabolic Effects of Non-Nutritive Sweeteners. Physiology \& Behavior, 152, 450-455. https://doi.org/10.1016/j.physbeh.2015.06.024

[39] Lustig, R.H., Schmidt, L.A. and Brindis, C.D. (2012) Public Health: The Toxic Truth about Sugar. Nature, 482, 27-29. https://doi.org/10.1038/482027a

[40] Fowler, S.P.G. (2016) Low-Calorie Sweetener Use and Energy Balance: Results from Experimental Studies in Animals, and Large-Scale Prospective Studies in Humans. Physiology \& Behavior, 164, 517-523.

[41] Yang, Q. (2010) Gain Weight by “Going Diet?” Artificial Sweeteners and the Neurobiology of Sugar Cravings: Neuroscience 2010. The Yale Journal of Biology and Medicine, 83, 101-108.

[42] Bazzano, L.A., Hu, T., Reynolds, K., Yao, L., Bunol, C., Liu, Y., et al. (2014) Effects of Low-Carbohydrate and Low-Fat Diets: A Randomized Trial. Annals of Internal Medicine, 161, 309-318. https://doi.org/10.7326/M14-0180 